\section{Decisiones y desafíos en un proceso de formación de posgrado \\ para la gestión en educación}

\author{
MILAGROS SOSA SÁLICO(1) \\ MARCELINO MAINA(2) \\ JULIA BERNIK(3) \\ NATALIA DÍAZ ${ }^{(4)}$
}

Fecha de recepción 29/05/18

Fecha de aceptación: 01/09/18
Resumen. En el presente artículo describimos y analizamos parte de un proceso de formación de posgrado realizado en el marco de un convenio entre la Universidad Nacional del Litoral (UNL) y el Ministerio de Educación Provincial (MEP): la Maestría en Políticas Públicas para la Educación (MPPE). Dicha Maestría tuvo como objetivo brindar marcos conceptuales y metodológicos vinculados con la gestión y el gobierno de las instituciones a diferentes escalas y niveles dentro del sistema educativo, centrados particularmente en la provincia de Santa Fe. Organizamos el análisis de la experiencia en cuatro apartados: el primero, retoma el encuadre general del surgimiento del proyecto de maestría; el segundo, describe momentos del diseño y la implementación de la segunda cohorte especialmente destinada a directivos del sistema educativo provincial; $y$, el tercero, describe el desarrollo de dos instancias propias de este proceso de formación: un Taller de Acompañamiento a la escritura de los Trabajos Finales y la Pasantía. Ambas resultaron relevantes en el apoyo a la producción académica y a la luz de la discusión sobre las prácticas de gestión. Finalmente se comparten reflexiones en torno a las implicancias de esta experiencia, que se consideran aportes para la mejora de un proceso de formación de directivos así como también para la optimización de políticas públicas compartidas entre la universidad y el MEP.

Palabras clave. experiencia de posgrado . acciones interinstitucionales · formación para la gestión escolar
(1) Docente e investigadora. Licenciada en Ciencia Política, egresada de la Facultad de Ciencia Política y Relaciones Internacionales de la Universidad Católica de Córdoba. Magíster en Administración Pública de la Universidad Nacional de Córdoba. En la actualidad se desempeńa como profesora en la cátedra de Introducción a la Ciencia Política y Trabajo Final de carrera. Dirige la Maestría en Políticas Públicas para la Educación en la Facultad de Humanidades y Ciencias de la Universidad Nacional del Litoral. Santa Fe, Argentina. milasosasalico@gmail.com (2) Docente e Investigador. Profesor en Historia por la Facultad de Formación Docente en Ciencias (actual FHUC) UNL. Magister en Ciencias Sociales. Estudios avanzados en Maestría en Docencia Universitaria. Doctorando en Estudios Sociales por la FHUC UNL. En la actualidad se desempeńa como profesor de la cátedra de Historia Argentina y en la Secretaría de Posgrado de la Facultad de Humanidades y Ciencias de la Universidad Nacional del Litoral. Su especialidad es la historia política y cultural argentina contemporánea. marcelinomaina@hotmail.com (3) Docente e investigadora. Profesora en Ciencias de la Educación (Facultad de Ciencias de la Educación FCEDU, Universidad Nacional de Entre Ríos uner). Magister en Didácticas Específicas (Facultad de Humanidades y Ciencias FHuc, Universidad Nacional del Litoral UNL). Doctora en Educación (FCEdu, Uner). Profesora adjunta ordinaria de Didáctica General (FHUC), integrante del Comité Académico de la Maestría en Políticas Públicas para la Educación (FHUC UNL). bernikjulia@gmail.com (4) Docente e investigadora. Profesora en Ciencias de la Educación, egresada de la Facultad de Ciencias de la Educación de la Universidad Nacional de Entre Ríos (FCEDU- UNER). Posee estudios de Especialista y Magíster en Educación. udEsA. Actualmente se desempeña en la Facultad de Humanidades y Ciencias y en la Secretaría de Planeamiento Institucional y Académico de la Universidad Nacional del Litoral. SantaFe, Argentina. diazsolnaty@gmail.com 


\section{Decisions and challenges in a postgraduate training process for education management}

\begin{abstract}
In this article, we describe and analyze part of a postgraduate training process carried out within the framework of an agreement between Universidad Nacional del Litoral (UNL) and the provincial Ministry of Education (MEP, for its Spanish initials): the Master's Degree in Public Policies for Education (MPPE). The objective of this Master's Degree was to provide conceptual and methodological frameworks linked to the management and governance of institutions at different scales and levels within the education system, particularly focusing on the province of Santa Fe. We organized the analysis of the experience into four sections: the first one takes up the general framework of the emergence of the master's degree project; the second section describes design and implementation features
\end{abstract}

of the second cohort, especially aimed at managers in the provincial education system; and the third one describes the development of two instances of this training process: a Final Work-Support Workshop and Internships. Both were relevant to academic production and in light of the discussion on management practices. Finally, reflections are shared around the implications of this experience, which are considered as contributions to both the improvement of a process of managerial training and the optimization of public policies shared by university and MEP.

Keywords. postgraduate experience . interinstitutional actions · training for school management
(1) Convenio Marco de Cooperación 29/3/12 aprobado por Ley provincial $\mathrm{N}^{\circ} \mathrm{I} 3.323$ y promulgada por Decreto No $4003 / 12$.

(2) Los proyectos y acciones se diseñaron como instrumento de definición de líneas de trabajo en el marco del Proceso de Programación del Plan de Desarrollo Institucional UNL 20IO-20I9. Se vinculan con los Objetivos del Plan y buscan mejorar una situación, crear una nueva realidad o solucionar una necesidad sentida o un problema existente.

\section{Inicios del proyecto, encuadre institucional}

En el marco de un Convenio interinstitucional entre el Ministerio de Educación de la provincia de Santa Fe (MEP) y la Universidad Nacional del Litoral (UNL) en el año $2012^{(1)}$ se comenzaron a realizar una serie de acciones entre las que se encuentra la Maestría en Políticas Públicas para la Educación (MPPE).

Ese mismo año, entre autoridades y docentes de la Facultad de Humanidades y Ciencias (FHUC) y la Secretaría de Planeamiento de UNL, se elaboró un Proyecto y Acción (en adelante PyA ${ }^{(2)}$ ) denominado «Formar para elaborar políticas públicas en educación» en consonancia con el inicio del Plan de 
Desarrollo Institucional 2010-2019(3) otorgando el marco político académico al proyecto de Maestría.

En sus fundamentos, el PyA afirma que la Maestría se cimienta y encuentra argumentos en preocupaciones intra e inter institucionales, presentes y acuciantes en toda la región y, en particular, a lo largo y ancho del territorio provincial. En lo que respecta a las primeras, fue intención de la Universidad incrementar la solidaridad intra-sistémica entre las diferentes áreas y organismos dependientes de la misma (Áreas Centrales, Unidades Académicas, instituciones pre-universitarias); reforzar el acompañamiento y habilitación a propuestas creativas e innovadoras y propiciar la participación de diferentes equipos docentes vinculados con las Ciencias Sociales y Humanas, en la formación de posgrado.

En lo que concierne a las preocupaciones interinstitucionales, la Universidad asumió el desafío de ofrecer nuevos espacios de formación que atendieran la complejidad que hoy conforman algunas prácticas profesionales específicas, en este caso, las relacionadas con la gestión y el gobierno de las instituciones educativas. Más allá de las tensiones que generalmente atraviesan todo proyecto de trabajo compartido, especialmente en lo que hace a sus definiciones políticas y metodológicas, en este caso y en el seno de los intercambios entre miembros de la UNL y del MEP, lo que se valora especialmente es la concreción del mismo a través de acciones mancomunadas con el órgano de coordinación educativa provincial central: el Ministerio de Educación de la Provincia, sus diferentes dependencias y áreas institucionales.

Son diversas las investigaciones( ${ }^{(4)}$, diagnósticos y estudios que advierten que lo realizado en materia de políticas públicas destinadas particularmente al campo educativo, pareciera no haber impactado satisfactoriamente en aquellas experiencias y prácticas que pretendían modificarse y mejorarse. Por el contrario, la persistencia de determinadas problemáticas -las que atañen a la gramática escolar, a la gestión de las instituciones educativas, a la constitución y sostenimiento colectivo de los proyectos institucionales, entre otras- estarían evidenciando la necesidad de revisar el sentido de estas políticas, por un lado, y por otro, las condiciones de apropiación y construcción de las mismas por parte de los sujetos a quienes van destinadas.
(3) La UNL tiene una larga trayectoria en la elaboración de Planes de Desarrollo, el primero fue construido en el ańo 2000 luego del primer proceso de Autoevaluación Institucional. El segundo PDi se diseñó en el año 20Io, al culminar la segunda Autoevaluación Institucional. El planeamiento es parte de una práctica arraigada en la institución que promueve la reflexión, el debate y participación de muchos actores no sólo en la definición de objetivos sino también en la producción de proyectos en las diferentes funciones sustantivas y de acompańamiento de la Universidad.

(4) A continuación, se citan algunos trabajos que dan cuenta de algunas perspectivas sobre el estudio de políticas públicas en educación en Argentina y particularmente en Santa Fe. Rivas, A. (2004) Gobernar la educación. Estudio comparado sobre el poder y la educación en las provincias argentinas. Granica. Buenos Aires. Ruiz, G. (20I2) Educación, politica y Estado. Definiciones y propuestas juridico-normativas de la política educacional. Editorial Académica Española. Ruiz, G. (20I3) «El enfoque jurídico normativo de la política educacional» en Tello, C. (coord. y comp.) Epistemologias de la politica educativa. Posicionamientos, perspectivas $y$ enfoques. Mercado de Letras. San Pablo. Sironi, M. (20I6) «La matriz política del gobierno de la educación santafesino. Acercamiento a normas y coyunturas críticas que configuraron el subsistema educativo en Santa Fe. 
En Mauro, S.; Ortiz de Rosas, V. y Vaca Narvaja, M. (comps.) Politica Subnacional en Argentina. Enfoques $y$ problemas. CEAP. UBA Sociales. Buenos Aires. Págs. 387 a 4I6. Sironi, M. (2017) «Democratizar el gobierno de la educación. Historia reciente y coyunturas críticas del subsistema educativo en Santa Fe». En Revista de la Escuela de Ciencias de la Educación, ańo I3, nro. I2, vol. 2, julio a diciembre de 20I7. Páginas I29-I47. ISSN I85I-6297. ISSN 2362-3349 (En Línea). Sironi, M.; Alasino, N. y Pezzetta, S. (2OII) «El rol del Estado en el ámbito educativo en la Provincia de Santa Fe». Ix Jornadas de Sociología. Facultad de Ciencias Sociales, Universidad de Buenos Aires, Buenos Aires.
En línea con estas consideraciones, la UNL decidió generar un nuevo proyecto institucional que implicó, por sobre todo, creatividad política (cfr. Frigerio, G., 2010). Y es por esto que se pensó una formación académica y profesional que habilitase otros recorridos para imaginar y decidir otras prácticas en la gestión y el gobierno de las instituciones educativas. La tarea exigió no solamente el abordaje de perspectivas teóricas y corpus categoriales específicos sino el diseño de mecanismos e itinerarios que lo hicieran posible, tornando fértiles los aportes teóricos a la luz de las experiencias y dando lugar a la emergencia de alternativas.

\section{Diseño e implementación de la propuesta}

En el año 2013 las Resoluciones № 010/13 del Consejo Directivo de la Facultad de Humanidades y Ciencias y la № 150/13 del Consejo Superior de la UNL dan marco institucional a la creación de la carrera y el 16 de diciembre de 2013 se celebra el Convenio Marco con el MEP que sella el acuerdo interinstitucional para dar lugar al inicio de la primera cohorte de la Maestría en Políticas Públicas para la Educación, en el año 2014. En esa ocasión, luego de lo sugerido por el Comité de Admisión a la carrera, se conformó la primera cohorte integrada por 44 profesionales provenientes de diversas localidades de toda la provincia de Santa Fe y también de Entre Ríos. Gran parte de los admitidos eran graduados de carreras universitarias y se desempeñaban en diferentes cargos y funciones en el Ministerio de Educación (Supervisores, Jefes de Supervisión y/o integrantes de equipo técnicos a cargo de programas). También conformaron la cohorte profesionales de la UNL responsables o con funciones en diferentes áreas de gestión institucional. Entre el año 2016 y 2017 egresaron 33 maestrandos y maestrandas. Durante el mes de agosto de 2016 se desarrolló la primera instancia de evaluación de 27 trabajos finales. Para la defensa oral, las presentaciones se agruparon de acuerdo a temáticas de investigación afines.

Fueron diversos los objetos de análisis que se abordaron en las diferentes producciones. Entre otros, los siguientes: calidad e inclusión educativa, 
formación docente, educación rural, la transmisión en educación, los intersticios entre salud y educación, las aulas como escenas teatrales, la decisión y responsabilidad, estudios históricos de los procesos de cambio de los diferentes niveles y modalidades del sistema educativo y estudios de casos sobre creación de escuelas universitarias.

La relevancia de la propuesta político educativa desarrollada por la FHUC UNL y el MEP en esta carrera de posgrado, se ve reflejada tanto en su diseño curricular como en la implementación del mismo. La carrera es de tipo profesional(5), de carácter estructurado, con modalidad presencial, de cursada intensiva y por cohorte cerrada.

Su diseño ofrece una variedad de instancias de formación conformadas en Módulos, los cuales pueden adoptar diversos formatos: seminarios, ateneos, debates, conferencias, entrevistas, sesiones de presentación de investigaciones, estudio y desarrollos metodológicos. Una instancia particular la configuran los denominados «Seminarios de Escribientes» a partir de los cuales los cursantes presentan los avances en sus proyectos de trabajo final y acreditan por tramos lo cursado. El diseño curricular también prevé la realización de un Seminario Internacional y una Pasantía. Esta última se propone como otra posibilidad de revisar y analizar corpus teóricos desarrollados a la luz de una inserción específica en espacios de gestión concretos, diferentes a los que cada maestrando y maestranda ocupaba habitualmente.

Las instancias de formación combinaron trabajos de profundización de temáticas abordadas por grupos de investigación provenientes de diferentes campos disciplinares; el desarrollo de corrientes de análisis particulares; la presentación y el estudio de casos y la consideración de programas y/o proyectos específicos de políticas públicas. Estos espacios de formación propiciaron la transversalidad disciplinar ${ }^{(6)}$ en los abordajes de la gestión y el gobierno de la educación como problema político y de políticas públicas. Se invitó a participar en diferentes Seminarios a diversos actores del sistema educativo, esto permitió el intercambio profundo y sistemático entre experiencias y debates teóricos-metodológicos.

La culminación del trayecto académico se completó con la presentación del Trabajo Final que podía conformarse desde diversas modalidades tales como: un análisis conceptual; una investigación exploratoria; un análisis
(5) cfr. Res Min. No I60/II y Resolución CS UNL No $414 / \mathrm{I} 2$.

(6) A modo de ejemplo, el dictado y coordinación de los seminarios La enseñanza como problema politico (Resolución CD No 223/18) y La evolución de las preguntas: ensayos de respuestas (Resolución $\mathrm{CD}$ № $38 \mathrm{I} / \mathrm{r} 8$ ) estuvo a cargo de docentes investigadores del campo de la Arquitectura, la Semiótica, la Historia, la Pedagogía y la Didáctica. 
(7) Convenio MEP - UNL 20I7; Res. CS No 95/I7.

(8) Pueden consultarse los programas correspondientes a cada uno de los Módulos (Res. CD FHUC No I75, 223, 285, 370, 427, 459,55I, 304,339, 38I, 443, 499 del ańo 20ı7). de caso; una indagación sobre prácticas institucionales y profesionales o el diseño de un proyecto de acción o intervención Luego de esta primera experiencia, la FHUC y el MEP celebran, en 2017, un segundo convenio con el objetivo de continuar la tarea de formación para la gestión pero con la particularidad de focalizar como destinatarios a integrantes de equipos directivos que se desempeñan en diferentes niveles del sistema educativo. En el convenio, se pauta una determinada cantidad de plazas destinadas a equipos directivos elegidos por el MEP y otra cierta cantidad de postulantes cuyas solicitudes fueron evaluadas por el Comité de Admisión, integrado por académicos de FHUC y miembros designados por el MEP. Respecto de las plazas seleccionadas por el MEP, fueron asignadas teniendo en cuenta como criterio de selección, además de la consideración de antecedentes de los postulantes, la representatividad equitativa de equipos de instituciones educativas de gestión pública y privada de diferentes regiones de la provincia. ${ }^{(7)}$

Nuevamente, y al igual que en la primera cohorte, están representadas todas las regiones y niveles educativos de la provincia, aunque predominan en cantidad aquellos provenientes del nivel inicial y primario (más del $50 \%$ de los admitidos). Como rasgo a destacar para este caso indicamos que, una parte significativa del grupo (egresados de Institutos de Formación Docente), experimenta por primera vez el ingreso a una carrera de formación en el sistema universitario.

Para acompañar el proceso de formación de esta cohorte se tomaron nuevas decisiones con la intención de enriquecer la propuesta curricular de la carrera y su funcionamiento académico. Así, optimizando la disposición flexible del diseño curricular vigente, se abrieron espacios de análisis específicos sobre planeamiento educativo y gestión escolar; trayectorias educativas; formatos y gramática escolar y la dimensión pedagógico-política del trabajo de los equipos directivos entre otros tópicos de relevancia( ${ }^{(8)}$. También, y en virtud de acuerdos del MEP con la Universidad Autónoma de México y la Universidad de Tempere de Finlandia, se contó con la presencia de docentes investigadores que dictaron seminarios e intercambiaron relatos sobre experiencias de formación (Resoluciones CD FHUC N 495 y 499 de 2016). Respecto del funcionamiento académico de la carrera, el nuevo convenio 
incorporó la figura del Gabinete ${ }^{(9)}$, conformado en forma mixta con docentes de FHUC y funcionarios del MEP, para acompañar la gestión realizada por el Comité Académico(10) de la carrera, y amplió la cantidad de coordinadores académicos. Las instancias de formación estuvieron a cargo de docentes-investigadores y extensionistas de la Universidad Nacional del Litoral, la Universidad Nacional de Entre Ríos y la Universidad Nacional de Rosario, conservando además, tal y como se mencionara anteriormente, la mirada de varias disciplinas para el abordaje de los asuntos de gestión educativa. Asimismo, se planteó la participación de egresados y egresadas de la primera cohorte con el objetivo de relatar sus experiencias respecto a la elección y desarrollo de las temáticas de los trabajos finales, muchos de los cuales comenzaron a participar en la dirección y/o codirección de los trabajos finales de la segunda cohorte.

En relación con las implicancias en la elaboración de estos trabajos finales, los maestrandos y las maestrandas expresaron la necesaria intervención del Equipo de Coordinación Académica para generar otras instancias de profundización en la escritura académica durante el posgrado. Es por ello que atendiendo este requerimiento, y a la luz de las modificaciones y mejoras que ya se habían empezado a implementar con esta cohorte, se revisó el sentido y la forma de cumplimiento de la Pasantía; se incorporaron otras instancias de formación en metodología de la investigación y se diseñó e implementó un Taller de Acompañamiento a la Escritura Académica del Trabajo Final que estuvo a cargo de docentes integrantes del Comité Académico de la Maestría. Entendiendo que éste constituye un espacio medular en la formación y directamente vinculados con la producción escrituraria del trabajo final de posgraduación.

\section{El Taller de Acompañamiento a la escritura de TF y la Pasantía}

Quienes transitamos los espacios académicos conocemos la intensidad que atraviesa a todo proceso de producción de un escrito, cualesquiera sean sus razones y marcos que le dan origen. El ejercicio de reunir en un escrito
(9) El Gabinete quedó integrado por: Mg. Laura Corral, egresada de la rera cohorte, Directora de la Escuela de Nivel Inicial y Primario de UNL y miembro del equipo de coordinación académica de la 2 da cohorte, la Dra. Silvia Morelli por el MEP y la directora de la carrera, Mg. Milagros Sosa Sálico (FHUC-UNL).

(10) El Comité Académico de la 2 da cohorte se conforma con: El coordinador académico Mg. Marcelino Maina (FHUC-UnL), la Mg. Natalia Díaz (FHuc-unL), la Dra. Julia Bernik (FHUC-UNL), la Dra. Silvia Morelli por el MEP y la Directora de la carrera. A fin de completar el equipo de trabajo, se suman en el acompańamiento a la coordinación académica las representantes del MEP: Mg. Erica Iturbe y $\mathrm{Mg}$. Marta Crivelli. 
(11) $\mathrm{Al}$ inicio de este tercer semestre, los y las cursantes entregaron y socializaron sus primeros trabajos escritos vinculados con el trabajo final,

los que fueron presentados durante el denominado Seminario de Escribientes I. En dicho Seminario, y desde una mecánica de intercambio horizontal, entre pares y con la presencia y valoraciones de un grupo de académicos externos a la Maestría, cada maestrando y maestranda expuso sus avances a consideración de todo el grupo. En la oportunidad de este Taller de acompañamiento, se les solicitó expresamente que partieran de estos escritos con el sentido de fortalecer $y / o$ rectificar, retomar y reconstruir el proyecto. académico claro y a la vez profundo las complejas tramas de reflexión, de prácticas, de experiencias que lo acompañan y/o preceden, suele tornarse un proceso difícil y exigente a la vez que convocante. $Y$ requiere algunas condiciones casi excluyentes: espacios y tiempos reales que lo tornen posible. La escritura del Trabajo Final (TF) en la MPPE, particularmente para esta segunda cohorte, se configuró en una inquietud central para el equipo de coordinación. Desde la consideración en torno a las complejas condiciones materiales y simbólicas en las que se desenvuelven las prácticas cotidianas que como directivos desarrollan los y las estudiantes de este posgrado, se atendió con mayor cuidado la habilitación de procesos reflexivos desde las diferentes instancias de cursado, de manera que sean ocasiones valiosas para ir pensando y estructurando aspectos vertebradores del TF. Así, y como figuraba en los marcos institucionales que dan origen a la Maestría, el desafío fue propiciar ocasiones para que, durante el cursado, puedan ir revisando sus itinerarios de prácticas de gestión como directivos de instituciones educativas, a la luz de los diferentes aportes y experiencias ofrecidas. Analizar sentidos, interpelar decisiones y acciones, reconocer aciertos y, en esa dinámica reflexiva, ir construyendo un posible objeto de estudio para sus Trabajos Finales.

Entonces, se procuró que durante los Seminarios los profesores y coordinadores posibiliten efectivamente estos cruces y, en este marco de acciones, se incorporó un nuevo espacio de trabajo que se denominó Taller de Acompañamiento a la escritura de los Trabajos Finales.

El Taller se ofreció durante el tercer semestre de 2018 por considerarlo un momento propicio para articular, con mayor rigurosidad, las vicisitudes de las prácticas cotidianas con los aportes teóricos. Se estructuró en tres momentos de intercambio, los que fueron precedidos por ejercicios de escritura en los que debían 'revisitar' sus primeros escritos para comenzar a focalizarse en pos del trabajo final de carrera(11). Se les sugirió presentar sus primeras precisiones respecto de un posible tema de investigación, preocupaciones iniciales, razones y justificación, selección de corpus categorial inicial y ámbito disciplinar de referencia para dicho estudio. Este ejercicio de producción permitió organizar con mayor claridad los ejes de los tres momentos que conformaron el Taller, los que se desarrollaron durante los 
meses de mayo y junio, cada veinte días. Se procuró entonces ofrecer espacios que les permitieran volver sobre estas nuevas consideraciones y trabajar en torno a ellas.

En el primer momento, se presentó el sentido de la escritura de TF en el contexto de un proceso de formación como directivos de instituciones educativas. Se reflexionó en torno a los diferentes ángulos y dimensiones que nutren el proceso de construcción de un problema susceptible de ser estudiado en un trabajo de investigación o abordado desde un trabajo de intervención ${ }^{(12)}$. Se focalizó especialmente en las implicancias de la objetivación, como práctica necesaria de reflexión y construcción teórica.

En el segundo momento se estudiaron las particularidades y semejanzas entre un trabajo de investigación y un trabajo de intervención institucional. Aquí se puso especial atención en dirimir diferencias y alcances de un trabajo académico, procurando ofrecerles ángulos de análisis que les permitieran pensar pertinencia y factibilidad para desarrollar uno $u$ otro de acuerdo a sus inquietudes, avances y condiciones de producción. En esta oportunidad también se invitó a colegas y especialistas para que relaten sus propias experiencias de escrituras bajo una u otra modalidad.

En el último momento, se realizó un análisis en torno a las implicancias del escrito final, insistiendo especialmente en la importancia de distinguir el proceso de escritura durante el desarrollo del trabajo en sí del proceso de escritura del informe final. Se centró en la importancia de reconocer lógicas de pensamiento, tiempos de reflexión y análisis diferentes aunque sustancialmente articulados. Por ello se abordó el sentido del plan de trabajo como hipótesis inicial; el avance en lecturas y/o trabajo de campo, según los casos; el ejercicio permanente de hacer dialogar estos avances con los presupuestos iniciales; la práctica de sistematizar, de ordenar análisis, reconocer hallazgos y, finalmente, escribir el texto final.

Los tres momentos se desarrollaron desde la metodología de Taller, para lo cual previamente a cada instancia, se les proponían una serie de lecturas de materiales bibliográficos diferentes que enriquecieron la discusión durante el desarrollo de los mismos. Cada momento ofreció como foco de análisis aspectos y cuestiones que los y las cursantes expresaron en el trabajo que inicialmente se les había solicitado. Si bien los tres momentos del Taller no
(12) Artículo 28 del Reglamento vigente de la carrera de posgrado. 
fueron planteados como obligatorios ni acreditables, la asistencia a los mismos fue casi completa.

Durante el cuarto y último semestre se propuso otra instancia de reflexión en torno a los procesos de estructuración de TF, la que tuvo como rasgo central la habilitación del trabajo entre pares. En tal sentido, el espacio curricular denominado Pasantía se organizó como ocasión para el intercambio y la evaluación compartida de nuevas escrituras que reunieron los avances sustantivos en cada proceso. Por ello se les propusieron una serie de orientaciones para realizar el escrito y organizar el intercambio entre pares, las que también pautaban tiempos y criterios de lectura.

Respecto del escrito, se les solicitaba especialmente brevedad, claridad y rigurosidad en la presentación del avance de manera de facilitar el diálogo con el o la colega, atendiendo a lo acotado de los tiempos y espacios disponibles para ello. Una vez recibidos los trabajos escritos el equipo de coordinación académica organizó las parejas de lecturas según temáticas que se consideraban afines y reenvió los mismos a los grupos correspondientes vía correo electrónico. Cada maestrando o maestranda, una vez recibido el escrito, realizaba su lectura e intercambiaba sus valoraciones entre colegas, las que se sistematizaban también por escrito. Luego del intercambio, se enviaban a la coordinación académica ambos escritos.

Se trató de otra instancia de interlocución acotada, delimitada pero sustancialmente valiosa que, junto con el resto de las instancias, se propuso para fortalecer la comprensión y apropiación genuina de la diversidad de aportes teórico disciplinares ofrecidos desde constantes espacios de reflexión sistemática. Espacios que partieron de sus propias inquietudes y prácticas, de las vicisitudes de gestionar instituciones educativas hoy y persiguieron el sentido de propiciar escrituras académicas que se reconozcan, iluminen e intervengan en las complejidades de las instituciones escolares, y no sólo las describan. 


\section{Reflexiones finales}

En tiempos donde el funcionamiento de las instituciones en general es objeto de interpelaciones constantes por gran parte de los sectores que constituyen el tejido social actual, las instituciones educativas y la gestión aparecen, en particular, como «la punta de un iceberg» que esconde diversidad de problemáticas, cuya resolución no depende exclusivamente de un individuo o grupo a la vez que, exige miradas amplias sobre las configuraciones históricas, políticas, culturales, ideológicas, que las constituyen.

Consideramos que la experiencia de la Maestría en Políticas Públicas para la Educación permite que los maestrandos -directivos de escuelas- visibilicen ángulos relevantes de análisis sobre la complejidad de sus prácticas. Ángulos que habilitan la construcción de otras prácticas posibles aún en los escenarios institucionales cotidianos que, frecuentemente, están marcados por profundas dificultades y condicionantes. La concreción de este posgrado nos persuade de la idea que es posible sostener un trayecto de formación para directivos de instituciones educativas de diferentes niveles del sistema cuando se habilitan canales interinstitucionales (UNL-MEP) para la decisión, intervención sistemática y sostenimiento académico, simbólico y material de dicho trayecto.

Así también reconocemos la relevancia de ofrecer, desde estos marcos institucionales que lo posibilitan, itinerarios de formación en la práctica profesional que insisten en la relevancia de pensar, analizar, leer, interpelar teorías desde la dinámica en que estas prácticas devienen cotidianamente. Poner en entre dichos las teorías desde las urgencias de las prácticas de gestión y gobierno escolar. Creemos que puede tornarse quimérico un proyecto de formación que se proponga transformar las prácticas sin reconocer como condición de ese pensar a la reflexión sobre los modos de pensar y hacer esa experiencia, en este caso la de gestionar instituciones escolares. Esta condición es indispensable para imaginar otras instituciones y concretar decisiones. Con este sentido se propusieron estos nuevos itinerarios de formación pensados para esta segunda cohorte de la MPPE. Estimamos que los mismos no sólo redundaron en la mejora y relevancia pedagógica para el cursado sino y sobre todo fortalecieron la mirada sobre el lugar del director y la directora desde posiciones autorreflexivas sobre las prácticas de gobierno y gestión escolar como prácticas políticas, pedagógicas y humanas. 


\section{Referencias bibliográficas}

Edelstein G. (2010). Enseñanza, políticas de escolarización y construcción didáctica. En Frigerio G. Diker G. (2010) Educar: ese acto político. Paraná. Editorial Fundación La Hendija.

Frigerio G. (2010). Educar: ese acto político. Paraná. Editorial Fundación La Hendija.

Saleme M. (1997). Una relación casi olvidada: el docente y el conocimiento. Córdoba. Editorial Decires, Narvaja.

Schon D. (1992). La formación de profesionales reflexivos. Hacia un nuevo diseño de la enseñanza y el aprendizaje de las profesiones. Barcelona. Editorial Paidós.

Terigi F. (2004). La enseñanza como problema político. En Frigerio G., Diker G. (2004) La transmisión en las sociedades, las instituciones y los sujetos. Un concepto de la educación en acción. Buenos Aires. Editorial Novedades Educativas.

\section{Documentos institucionales citados}

Convenio Marco de Cooperación 29/3/12 entre el Ministerio de Educación de la provincia de Santa Fe y la Universidad Nacional del Litoral aprobado por Ley provincial $N^{\circ} 13.323$ y promulgada por Decreto $\mathrm{N}^{\circ}$ 4003/12.

Convenio MEP - UNL 2017. Resolución del Consejo Superior UNL No 95/17.

FHUC UNL (2013) Reglamento de la carrera Maestría en Políticas Públicas para la Educación.

Resolución del Ministerio de Educación de la provincia de Santa Fe No 160/11.

Resolución del Consejo Superior UNL No 414/12.

Resolución del Consejo Directivo FHUC UNL N 010/13. Resolución del Consejo Superior UNL N 150/13.

Resoluciones del Consejo Directivo FHUC UNL No 175 , 223, 285, 370, 427, 459,551, 304,339, 381, 443, 499 del año 2017.

Resoluciones del Consejo Directivo FHUC UNL N 223 y 381 del año 2018.

Universidad Nacional del Litoral (2010) Plan de Desarrollo Institucional 2010-2019. Hacia la Universidad del Centenario. Santa Fe.

Universidad Nacional del Litoral (2012) Proyecto y Acción «Formar para elaborar políticas públicas en educación». Santa Fe. Mimeo. 